\title{
Dynamic modeling and simulation of trailer with compliance steering system
}

\author{
Wang Qingyun ${ }^{1}$,Wang Peng ${ }^{2}$,Chen Ning ${ }^{2 *}$ \\ ${ }^{1}$ College of Automobile and Traffic Engineering, Nanjing Forestry University, Nanjing, 210037, China \\ ${ }^{2}$ College of mechanical and electrical engineering, Nanjing Forestry University, Nanjing, 210037, China
}

\begin{abstract}
Stability of a vehicle and tire wear is affected by the movement inference between the tractor and the trailer when the trailer is turning. Therefore, to solve the problem, Compliant Steering (CS) system is used. The system makes the rear-wheel of the trailer steer. After establishing a dynamic model of the trailer and the CS system, the simulation result shows that the system has a smaller lateral force. This smaller force at the hinge point indicates movement inference is reduced and motion response is far better. Furthermore, this paper contains the analysis of the influence of the key parameters of CS system and the speed on the moving characteristics of the trailer.
\end{abstract}

\section{Introduction}

Rear wheel CS technique is a passive steering technique not requiring steering mechanism that utilizes the lateral force and elasticity of the wheel and the rear suspension when the vehicle turns respectively [1]. That is when compared with the active steering technology, which is simple in structure and without energy consumption [2]. Currently, the research is abundant on CS technology used in large-scale vehicle [3].

A full trailer is made up of a tractor and a trailer. It is totally different from a car in terms of handling stability. On a small curvature road and turning in high speed, the full trailer with over-steer characteristics is easy to lead to folding or swing tail (trailer swing) [4]. Although low speed, problem of movement inference between the tractor and trailer during steering exists, thus, aggravating tire wear. In the past, due to small number of trailer, attention was not given. Currently, high rural mechanization and traffic, the number of trailer and accident is increasing gradually.

Recently, many scholars are researching and focusing on the dynamic analysis of trailers. After establishing a dynamic model of the trailer and the compliant steering system, simulation analysis of the trailer result shows the CS technology can reduce movement interference between the tractor and the trailer, improving the stability of the vehicle.

\section{Dynamic Analysis of Full Trailer}

\subsection{Dynamic Equation of Articulated Full Trailer}

The articulated full trailer model is shown in Fig. $1[5,6]$. Here $F_{\text {yi }}(i=1 \sim 4)$ represents tire lateral force, $\delta_{1}$ is front wheel angle, $\Delta \varphi$ is the angle of deviation between the tractor and the trailer, $F_{\mathrm{Tx}}, F_{\mathrm{Ty}}$ are the longitudinal and lateral forces of the hinge. Other annotations are shown in Tab 1.

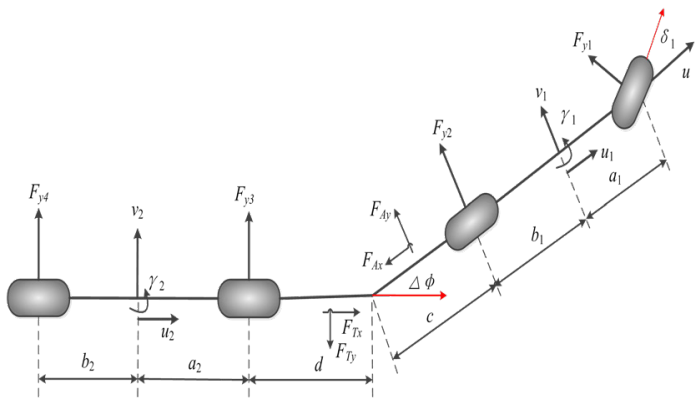

Fig.1. Mechanical analysis of the trailer when steering.

After applying a force-balance analysis [7] to the model in Fig.1, the dynamic equations can be derived. Then they are formulated in a standard state space form, thus

$$
\boldsymbol{A} \dot{\boldsymbol{X}}=\boldsymbol{B} \boldsymbol{X}+\boldsymbol{D q}
$$

where the state variable is taken as $\boldsymbol{X}=\left[\begin{array}{llll}\beta_{1} & \gamma_{1} & \gamma_{2} & \Delta \varphi\end{array}\right]^{T}, \beta_{i}$ is the vehicle side slip angle, $\gamma_{\mathrm{i}}$ is yaw rate $(i=1,2$ The tractor is labeled 1 and the trailer is 2), and $q$ is the linear tire [8] lateral force vector

$$
\begin{aligned}
& \boldsymbol{q}=\left[\begin{array}{llll}
F_{y 1}\left(\alpha_{1}\right) & F_{y 2}\left(\alpha_{2}\right) & F_{y 3}\left(\alpha_{3}\right) & F_{y 4}\left(\alpha_{4}\right)
\end{array}\right]^{T} \\
& \boldsymbol{A}=\left[\begin{array}{cccc}
m_{1} \cdot u \cdot\left(b_{1}+c\right) & I_{Z 1} & 0 & 0 \\
\left(m_{1}+m_{2}\right) \cdot u & -m_{2}\left(b_{1}+c\right) & -m_{2}\left(a_{2}+d\right) & m_{2} \cdot u \\
m_{1} \cdot u \cdot\left(a_{2}+d\right) & 0 & I_{Z 2} & 0 \\
0 & 0 & 0 & 1
\end{array}\right],
\end{aligned}
$$

\footnotetext{
* Corresponding author:chenning@njfu.com.cn
} 


$$
\begin{aligned}
\mathrm{B} & =\left[\begin{array}{cccc}
0 & -m_{1} \cdot u \cdot\left(b_{1}+c\right) & 0 & 0 \\
0 & -m_{1} \cdot u & -m_{2} \cdot u & 0 \\
0 & -m_{1} \cdot u \cdot\left(a_{2}+d\right) & 0 & 0 \\
0 & 1 & -1 & 0
\end{array}\right], \\
D & =\left[\begin{array}{cccc}
\left(b_{1}+c+a_{1}\right) & c & 0 & 0 \\
1 & 1 & 1 & 1 \\
a_{2}+d & a_{2}+d & a_{2} & -b_{2} \\
0 & 0 & 0 & 0
\end{array}\right] .
\end{aligned}
$$

\subsection{Model of trailer with rear wheel compliant steering}

From the dynamic analysis viewpoint, if the rear wheels of the trailer steer a small angle which is opposite to the turning direction [9], the stability of the trailer can be improved, and the lateral interference between the trailer and the tractor can be weakened [10, 11]. Therefore, the objective of the study is the full trailer with rear-wheel steering system. The CS system used here is shown in Fig.2, $c_{2}, d_{2}$ are its front and rear arm length, $\delta_{4}$ is trailer rear wheel CS angle, regardless of the quality of the CS system and the effect of moment of inertia.

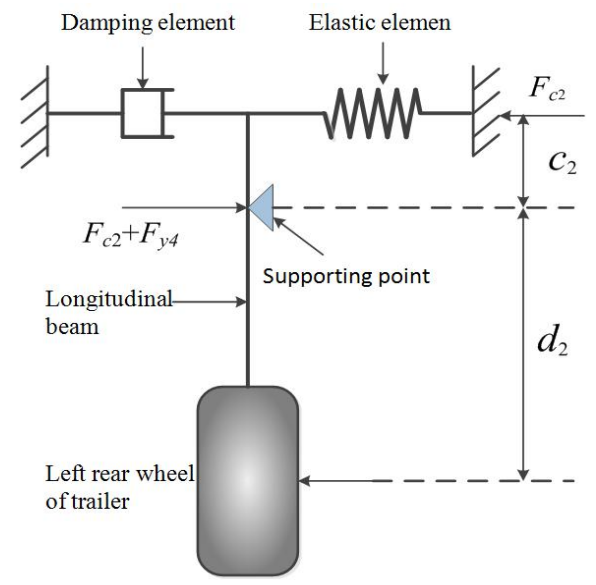

Fig. 2 Force diagrams of trailer CS system

$F_{\mathrm{C} 2}$ is the elastic and damping force of the CS combined, thus

$$
F_{c 2}=-k_{c} \cdot c_{2} \cdot \delta_{4}-c_{c} \cdot c_{2} \cdot \dot{\delta}_{4}
$$

The CS angle meets the following equation:

$$
\dot{\gamma}_{2}+\ddot{\delta}_{4}=-\frac{k_{c} d_{2}^{2}}{I_{c s 2}} \cdot \delta_{4}-\frac{c_{c} d_{2}^{2}}{I_{c s 2}} \cdot \dot{\delta}_{4}-\frac{c_{2}}{I_{c s 2}} \cdot F_{y 4}
$$

Let $\delta_{4}=\phi, \dot{\delta}_{4}=\omega$, take the state vector as $\boldsymbol{X}=\left(\begin{array}{llllll}\beta_{1} & \gamma_{1} & \gamma_{2} & \Delta \varphi & \phi & \omega\end{array}\right)^{T}$. The state equation is

$$
E \dot{X}=A X+N p
$$

$$
\boldsymbol{E}=\left[\begin{array}{cccccc}
1 & \frac{I_{Z 1}}{\left(b_{1}+c\right) m_{1} u} & 0 & 0 & 0 & 0 \\
1 & -\frac{m_{2}\left(b_{1}+c\right)}{\left(m_{1}+m_{2}\right) u} & -\frac{m_{2}\left(a_{2}+d\right)}{\left(m_{1}+m_{2}\right) u} & \frac{m_{2}}{\left(m_{1}+m_{2}\right)} & 0 & 0 \\
1 & 0 & \frac{I_{Z 2}}{\left(a_{2}+d\right) m_{1} u} & 0 & 0 & 0 \\
0 & 0 & 0 & 1 & 0 & 0 \\
0 & 0 & 0 & 0 & 1 & 0 \\
0 & 0 & 1 & 0 & 0 & 1
\end{array}\right],
$$$$
\mathbf{A}=\left[\begin{array}{cccccc}
0 & -1 & 0 & 0 & 0 & 0 \\
0 & -\frac{m_{1}}{m_{1}+m_{2}} & -\frac{m_{2}}{m_{1}+m_{2}} & 0 & 0 & 0 \\
0 & -1 & 0 & 0 & \frac{k_{c} d_{2}^{2}}{\left(a_{2}+d\right) m_{1} u} & \frac{c_{c} d_{2}^{2}}{\left(a_{2}+d\right) m_{1} u} \\
0 & 1 & -1 & 0 & 0 & 0 \\
0 & 0 & 0 & 0 & 0 & 1 \\
0 & 0 & 0 & 0 & -\frac{k_{c} d_{2}^{2}}{I_{c s 2}} & -\frac{c_{c} d_{2}^{2}}{I_{c s 2}}
\end{array}\right]
$$

$$
\mathbf{N}=\left[\begin{array}{cccc}
\frac{a_{1}+b_{1}+c}{\left(b_{1}+c\right) m_{1} u} & \frac{c}{\left(b_{1}+c\right) m_{1} u} & 0 & 0 \\
\frac{1}{\left(m_{1}+m_{2}\right) u} & \frac{1}{\left(m_{1}+m_{2}\right) u} & \frac{1}{\left(m_{1}+m_{2}\right) u} & \frac{1}{\left(m_{1}+m_{2}\right) u} \\
\frac{1}{m_{1} u} & \frac{1}{m_{1} u} & \frac{a_{2}}{\left(a_{2}+d\right) m_{1} u} & -\frac{b_{2}-c_{2}}{\left(a_{2}+d\right) m_{1} u} \\
0 & 0 & 0 & 0 \\
0 & 0 & 0 & 0 \\
0 & 0 & 0 & -\frac{c_{2}}{I_{c s 2}}
\end{array}\right]
$$

\section{Kinematics Simulation of Full Trailer}

The main parameters affecting the handling stability of the trailer in the CS system are [12,13],

- The stiffness of the CS system $k_{\mathrm{s}}$,

- the lengths of the front and rear arm $c_{2}$ and $d_{2}$, damping coefficient $C_{\mathrm{c}}$ or damping ratio $\zeta$,

- $\quad$ the speed of the vehicle $u$.

Regardless of the specific effects of these parameters, comparing with the vehicle and without CS system, the following Tab. 1 shows the specific parameter

Tab.1. Simulation parameters of a car

\begin{tabular}{cc}
\hline Parameter name & Values \\
\hline Tractor, trailer weight $m_{1}, m_{2}(\mathrm{~kg})$ & $6804 / 5443$
\end{tabular}

The longitudinal distance from the hinge point to

$$
\text { the rear axle of the tractor } c(\mathrm{~m})
$$

The longitudinal distance of the tractor center to the front axle and rear axle $a_{1}, b_{1}(\mathrm{~m})$

The longitudinal distance of the trailer center to the front axle and rear axle a2, b2 (m)

where 
The longitudinal distance from the front axle of the trailer to the hinge point $d(m)$

Trailer rear wheel elastic components, rear wheel center of mass to support distance $\mathrm{c} 2, \mathrm{~d} 2$ (m)

Trailer rear wheel CS stiffness $\mathrm{k} 2 \mathrm{~s}(\mathrm{n} / \mathrm{rad})$

45000

The damping ratio of CS system $\zeta$

Tractor, trailer around the z-axis of inertia IZ1、IZ2

$21693 / 125$

(kg.m)

Moment of inertia of the rear wheel CS system Ics2 (kg.m)

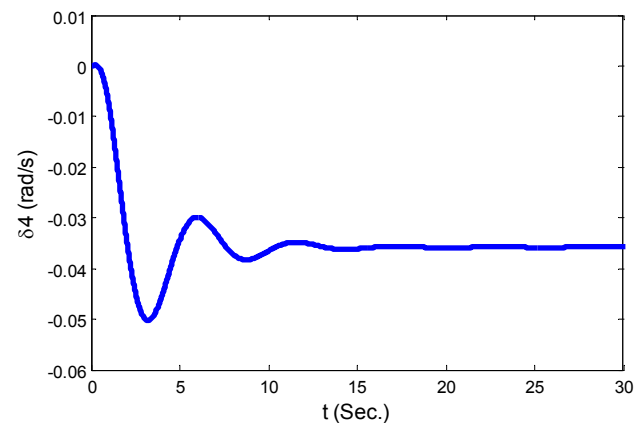

Fig.3. The curve of compliant rear-steering angle

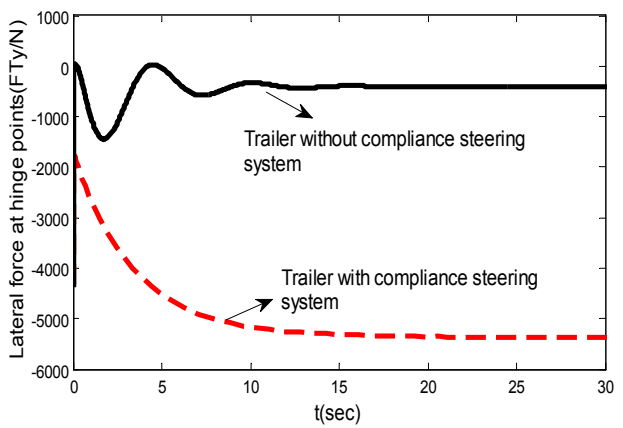

Fig.4. Lateral forces at hinge points

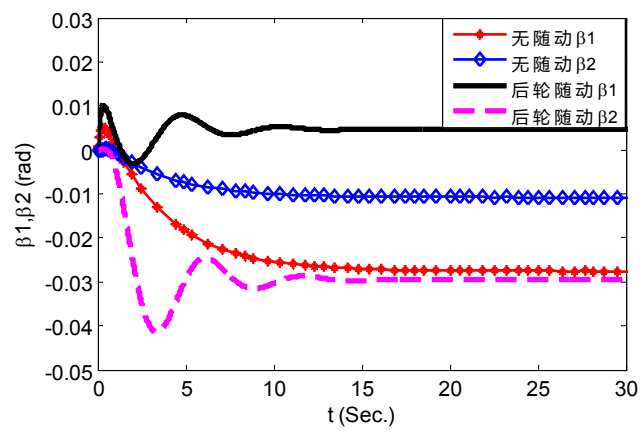

(a) The curves of side slip angle

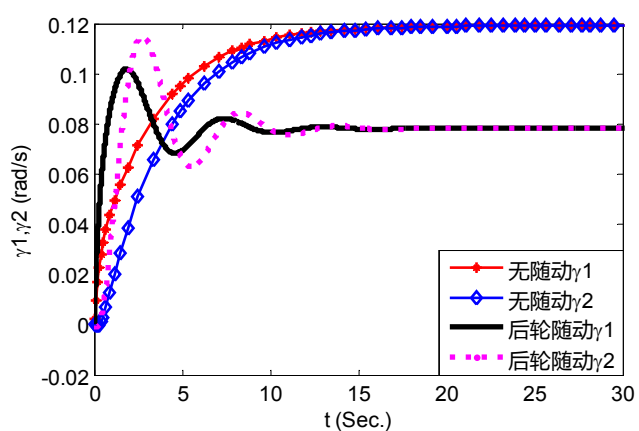

(b) The curves of yaw rate

Fig.5. Comparison of traditional trailer and trailer with CS system

When the vehicle with the CS turns, the rear wheels of trailer turn an angle opposite to the steering of the vehicle is shown in Fig.3. As shown in Fig.4, the generation of the compliant angle results in a significant decrease in the lateral forces at the hinge points. In Fig.5, it is visible that the yaw rate of the tractor and trailer and the side slip angle of the tractor are significantly improved.

The results verifies that when the rear wheel of trailer steers an angle, the lateral force between the tractor and the trailer is released, thereby reducing effectively the motion interference between the tractor and the trailer.

In order to analyze the specific characteristics of these parameters, packet simulation is required. Primarily, the effect of the sizes of $c_{2}$ and $d_{2}$ are discussed. Secondly, the influence of elastic element stiffness ks and damping ratio $\zeta$ is analyzed. Assuming that other parameters are the same, we only adjust the front and rear arm length $c_{2}$ and $d_{2}$. The values of the relevant parameters are: $u=10$ $\mathrm{m} / \mathrm{s}, \delta_{1}=0.02 \mathrm{rad}, \zeta=1, k_{\mathrm{s}}=45000 \mathrm{n} / \mathrm{m}$.

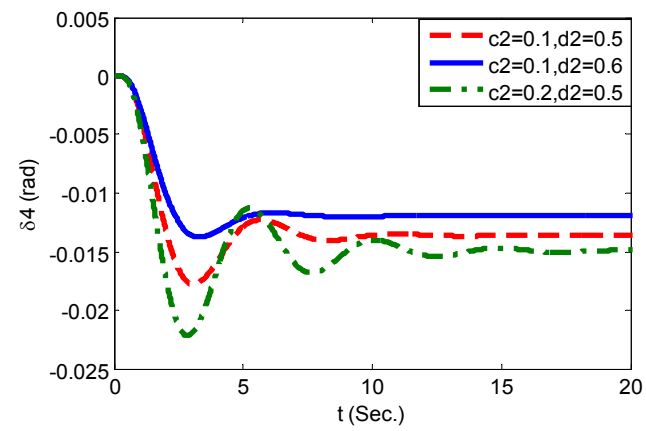

(a) The curves of compliant rear-steering angle 


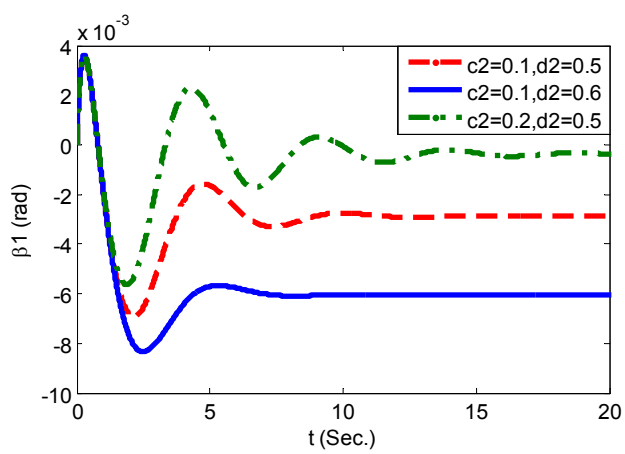

(b) The curves of side slip angle of tractor

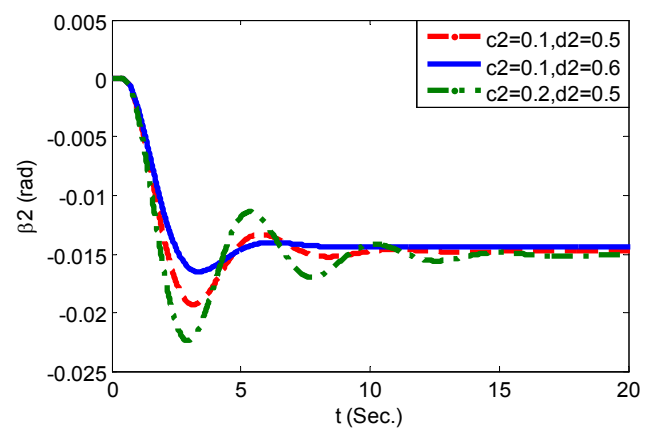

(c) The curves of side slip angle of trailer

Fig.7 The curves of compliant rear-steering

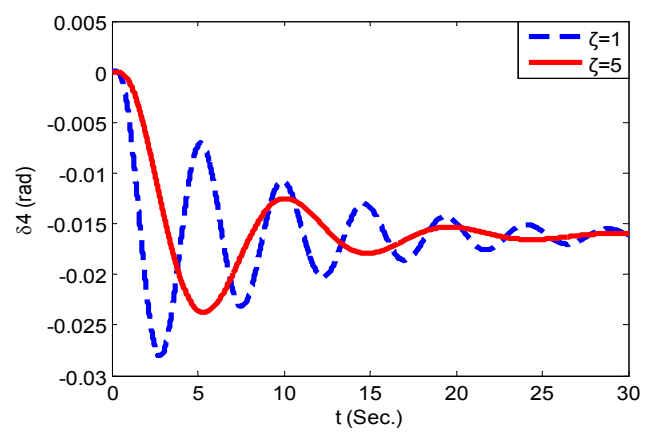

Fig.6. The curves when changing the support with different damping ratios point position angle

Theoretically, when the support point in the longitudinal beam on the relative position is declining, the same elastic force will produce a greater torque. As the relative position of the support point moves down (the value of $c_{2}$ increases and $d_{2}$ decreases relatively), the rear wheel angle of the trailer increases and the whole vehicle's vibration time and frequency are significantly increased. When the value of $c_{2}$ is greater than a certain value, the vehicle has been unstable. As shown in Fig 6, three sets of parameters are selected for comparison in the stable range. In Fig.6-a, when $c_{2}=0.1$ and $d_{2}=0.6$, it's compliant angle is relatively small, but its response is more stable. In Fig.6-b and Fig.6-c, the vehicle side slip angle is more stable, and the side angle of the tractor is relatively larger. When the parameter is chosen as $c_{2}=0.2$ and $d_{2}=0.5$, the system transient response is not good and the steady-state response is the best. Considering the effect of stiffness, we can refer to the dotted line in Fig.6-a and Fig.8-a as shown below. Through the design of specific parameters, these two CS angle response curves are obtained with the same parameters except the stiffness. With the increase of stiffness, the compliant angle response is more stable and the vehicle motion characteristics is improved. Fig.7 shows that when the damping ratio $\zeta=1$, the system has a long-time oscillation by adjusting the parameters, and then keeping the other parameters unchanged, we only increase the damping ratio, the oscillation time and amplitude of the system are significantly reduced. Although the damping ratio changes, the steady-state response of the rear wheel compliant angle unchanged. The final stability value of the side slip angles of the tractor and the trailer are not changed with the change of the damping ratio. It is clear that the stiffness and damping ratio of the compliant angle system are variable parameters in the moving system structure, and adjusting the values of both are more effective in improving the motion characteristics of the trailer.

At a low speed $(u=5 \mathrm{~m} / \mathrm{s})$, the CS angle of the trailer and the yaw rate of the tractor and the trailer is steady reached a fixed value. At this time tractor and trailer side angle is smaller. In the case of medium speed $(u=10 \mathrm{~m} / \mathrm{s})$, the side angle of the tractor and trailer increases. Those shows that a group of parameters of CS system is correspond to a certain speed of the vehicle, so that the performances are better relatively, and the increase in speed will reduce the handling of the full trailer stability. When the speed reaches a certain value, the vehicle becomes instable. When a more stable performance at higher speeds is required, adjust the parameters of the CS system according to the analysis above.

\section{Conclusion}

(1) When the rear wheels of trailer steers, the lateral forces of the tires are released, so the lateral force at the hinge point is smaller and the movement interference between the tractor and the trailer is reduced.

(2) Based on the theory of CS technology to study the stability of the full trailer, the dynamic analysis of the 
handling stability of the trailer with CS system is conducted. The specific impact of the various parameters and the speed on the performances of trailer are studied.

(3) The simulation results show that the angle of the rear wheel of the trailer can reduce the motion interference at the hinge point, and improve the stability of the vehicle, which is also consistent with the theoretical analysis. The key parameters of the CS system have a comprehensive effect on the vehicle, here the research methods and CS system parameters is provided for the study of the full trailer with compliant steering system.

\section{Acknowledgements}

This work was supported by the National Natural Science Foundation of China (Grant No. 11272159).

\section{References}

[1] Souharda R. Compliant Multi-link Vehicle Suspensions. South Carolina: Clemson University, 2008.

[2] Mu Xiangdong. Modeling and Test Verification of Citroen ZX Car Compliance Steering Characteristics. Journal of Highway and Transportation Research and Development, 2004, 21(9):127-130.

[3]Xu Xiaomei, Chen Ning, H. P. Lee. A Review on the Applied Research of Rear Wheel Compliance Steering. Automobile Technology, 2016, 7:1-6.

[4]Enomoto H, Akiyama K and Okuyama H. Advanced Safety Technologies for Large Trucks. In 14th Asia Pacific Automotive Conference, 2007.

[5]Yu Zhisheng. Automobile theory. Beijing: China Machine Press.

[6]Pacejka H B. Tire and Vehicle Dynamics (Second Edition). Burlington: Butterworth Heinemann, 2006.
[7]Wang Wei, Bei Shaoyi, Zhao Youqun, et al. Research on the development and future of vehicle handling and stability. Machine Design and Manufacturing Engineering, 2014, 43(10): 6-12.

[8]Wei Chaoyi, Chen Ke, Zhou Kongkang, et al. Stability Analysis of Operation for Passenger Car-trailer Based on Root-locus Method. Transaction of the Chinese Society for Agricultural Machinery, 2007,38(3): 15-18. [9]Kharrazi S, Lidberg M, Roebuck R, et al. Implementation of Active Steering on Longer Combination Vehicle for Enhanced Lateral Performance. Vehicle System Dynamics. 2012, 50(12): 1949-1970.

[10] Kristopher J Seluga RMOI. Articulated Vehicle Yaw Stability During Braking — A parametric Study. In Rosemout, lllinois USA, 2004.

[11]Wu D H, Lin J H. Analysis of Dynamic Lateral Response for a Multi-Axle-Steering Tractor and Trailer. Heavy Vehicle Systems-International Journal of Vehicle Design. 2003, 10(4):281-294.

[12]Rangavajhula K, Tsao H. Effect of Multi-axle Steering on off-tracking and Dynamic Lateral Response of Articulated Tractor-trailer Combinations. International Journal of Heavy Vehicle Systems. 2007, 14(4): 376-401.

[13]Tica M, Dobre G, Mateescu V, et al. Influence of Compliance for an Elasto-kinematic Model of a Proposed Rear Suspension. International Journal of Automotive Technology, 2014, 15(6): 885-891.

[14]Yang Xiujian, Li Yaoping, Xiong jian. Analysis on the Lateral stability and Instability Mechanism of Tractor Semi-trailer Combination. Automobile Engineering, 2011, 33(06): 486-493.

[15]Bai Bin. Study on Lateral Control ability and Stability of Tractor Trailer. Jilin: Jilin University. 